\title{
Plasma lipoproteins in visceral leishmaniasis and their effect on Leishmania-infected macrophages
}

\author{
N. M. SOARES, ${ }^{1}$ T. F. LEAL, ${ }^{1,2}$ M. C. FIÚZA, ${ }^{1,2}$ E. A. G. REIS, ${ }^{2}$ M. A. L. SOUZA, ${ }^{1}$ W. L. DOS-SANTOS ${ }^{2}$ \\ $\&$ L. PONTES-DE-CARVALHO ${ }^{2}$ \\ ${ }^{1}$ Departamento de Análises Clínicas e Toxicológicas, Faculdade de Farmácia, Universidade Federal da Bahia, Salvador, Brazil, ${ }^{2}$ Centro de \\ Pesquisa Gonçalo Moniz, Fundação Oswaldo Cruz, Salvador, Brazil
}

\section{SUMMARY}

This work aimed at investigating the lipid profile of zoonotic visceral leishmaniasis (VL) patients' sera and the effect of lipoproteins on the in vitro production of tumour necrosis factor- $\alpha(T N F-\alpha)$, interleukin $(I L)-6, I L-10$ and IL-12 by Leishmania infantum-infected and uninfected macrophages. Lipids were quantified in 26 VL patients' sera and 26 healthy controls from a VL endemic area. The patients' sera had higher triglyceride and very low density lipoprotein (VLDL) levels, and much lower apolipoprotein A1, total cholesterol, low density lipoprotein ( $L D L)$ and high density lipoprotein $(H D L)$ levels than the control sera. Lipoprotein fractions were obtained by ultracentrifugation of sera. The addition of LDL and HDL to Leishmania-infected and uninfected macrophages, in physiological concentrations, enhanced the production of IL-6 and IL-10, but not of IL-12. LDL stimulated the production of TNF- $\alpha$ only in infected macrophages, whereas HDL stimulated the production of lower amounts of TNF- $\alpha$ in both infected and uninfected macrophages. VLDL stimulated only the production of IL-10. It is proposed herein that LDL may influence the development of $V L$ by promoting the production of $T N F-\alpha$ by infected macrophages. A decrease in plasma LDL in some VL patients (to $20 \mathrm{mg} / \mathrm{mL}$ or less); however, would tend to reduce the production of $T N F-\alpha$ and therefore to limit the development of immune-mediated pathology, not withstanding the fact that it would perhaps increase the permissiveness of macrophages to Leishmania growth.

Keywords cytokines, Leishmania, lipoproteins

Correspondence: Neci Matos Soares, Departamento de Análises Clínicas e Toxicológicas, Faculdade de Farmácia, Universidade Federal da Bahia, Rua Barão de Geremunbo S/N, Ondina, CEP40170115, Salvador, Bahia, Brazil

(e-mail: neci@ufba.br).

Received: 5 August 2009

Accepted for publication: 3 November 2009

\section{INTRODUCTION}

Zoonotic visceral leishmaniasis (VL) is a systemic infectious disease caused by the protozoa of the Leishmania genus, which infect mononuclear phagocytes in the spleen, the liver and the bone marrow of human beings and other mammals (1). When left untreated, most patients die of opportunistic bacterial infections (2) because of a diseaseassociated nonspecific immunodeficient state (3-5). Zoonotic VL is caused by Leishmania infantum Nicolle, 1908, or Leishmania chagasi Cunha \& Chagas, 1937, which are believed to be the same parasite (6). In the American continent, it is transmitted by an insect vector of the genus Lutzomia $(7,8)$.

Some studies on both zoonotic VL and anthroponotic (caused by Leishmania donovani) VL have demonstrated that the control of the disease is associated with Th1-type cellular immune responses, with production of interleukin (IL)-12 and interferon- $\gamma$ (IFN- $\gamma$ ) (9-12). IL-12 stimulates the production of IFN $-\gamma$ in VL patients who respond well to treatment (9) and its plasma levels are lower in L. donovani-infected individuals with overt VL than in L. donovani-infected individuals who develop skin delayed-type hypersensitivity reactions to Leishmania antigens without developing a disease (11). Moreover, peripheral blood mononuclear cells (PBMC) from individuals with asymptomatic or self-healing oligosymptomatic L. infantum infection, and not from individuals that eventually develop progressive VL, respond in vitro to Leishmania antigen with the production of IFN- $\gamma$ and IL-2 (9). When the disease is already established; however, increased levels of the pro-inflammatory cytokines IFN- $\gamma$, tumour necrosis factor- $\alpha$ (TNF- $\alpha$ ), IL-6 and IL- 8 can be found in VL patients' plasma (13-16). Despite the increase in IFN- $\gamma$ and TNF- $\alpha$ plasma levels during active disease, the numbers of TNF- $\alpha$-containing monocytes (16) and the production of IFN- $\gamma$ by antigen-stimulated PBMC (15) are reduced when compared with that in 
asymptomatic and cured individuals respectively. Moreover, in $L$. infantum- or $L$. donovani-infected mice and human beings, the development of disease occurs in the presence of increased amounts of IL-10, which is a potent immunosuppressive and anti-inflammatory cytokine (15-19).

Lipoproteins or lipoprotein particles are capable of modulating cellular immune responses. Among these are chylomicrons, very low density lipoproteins (VLDL) and low density lipoproteins (LDL), which inhibit the proliferation of lymphocytes in vitro $(20,21)$. Macrophages, which are the cellular hosts of Leishmania parasites, have natural or modified lipoprotein receptors (22) and are therefore possible targets for the immunomodulatory activities of lipoproteins.

Plasma lipid alterations have been demonstrated in some infectious diseases. In acquired immune deficiency syndrome (AIDS), levels of serum triglycerides are elevated and those of high density lipoprotein (HDL) are decreased $(23,24)$. On mansonic schistosomiasis, the plasmatic concentration of total cholesterol and triglyceride levels are found to be decreased (25). In canine VL, high levels of triglycerides and low levels of HDL have been described (26). Alterations in circulating lipids in a low number of VL human patients have also been reported (27-30). Interestingly, several recent reports have described that depleting cholesterol from host-cell membranes impairs the penetration of intracellular pathogens, such as Mycobacteria, Plasmodium falciparum, Chlamydia trachomatis, Listeria monocytogenes, L. donovani and L. infantum (31-36). These findings have led to the proposition that cholesterol-depleting medications could play a therapeutic role in VL (37). On the other hand, the reduction in cholesterol concentrations in antigen-presenting cell membranes has been shown to impair antigen presentation to lymphocytes (38), a phenomenon that could theoretically lead to sub-optimal immune responses, and, therefore, favour parasitism. Studies on plasma lipid abnormalities in VL patients, and on the possible effect of the addition of these plasma lipoproteins to Leishmania-infected macrophages, therefore, may provide important clues on the role of these lipoproteins in the development of the infection.

In this report, the nature of the lipidic abnormalities in 26 VL patients' sera is described. In addition, the effects of lipoproteins on the in vitro production of pro-inflammatory and regulatory cytokines by $L$. infantum-infected and uninfected macrophages are reported, and a parallel between the lipoprotein concentrations found in the patients, and how these concentrations affect the cytokine production by infected and uninfected macrophages in vitro, is made.

\section{MATERIALS AND METHOD}

\section{Serum samples}

Samples of sera from 26 parasitologically diagnosed patients with acute VL were obtained at the Unity of Endemic Diseases Pirajá da Silva in Jequié (PIEJ), Bahia, Brazil. Blood samples for serum preparation were collected before chemotherapy. Control sera from 26 apparently healthy, age- and gender-matched individuals, who did not have detectable anti-Leishmania antibody levels in an ELISA (39), were obtained at the same region. All patients and volunteers were previously informed of the nature of the research and they agreed to participate in the study. The project was approved by the Committee of Ethics in Research of the Gonçalo Moniz Research Centre.

\section{Parasite culture}

Leishmania infantum was characterized by a panel of species-specific monoclonal antibodies and isoenzymatic patterns, by Dr. G. Grimaldi, at the Oswaldo Cruz Institute, Oswaldo Cruz Foundation, Rio de Janeiro, Brazil. Promastigotes were cultured in Schneider's medium supplemented with $10 \%$ foetal bovine serum (Microbiology, Rio de Janeiro, Brazil).

\section{Quantification of plasma lipids}

The serum amounts of triglycerides, cholesterol and fractions and apolipoproteins were determined with the use of commercially available kits (Labtest Diagnostica S.A., Belo Horizonte, Brazil), using enzymatic methodologies or Trinder's final (quinoneimine formation) reaction.

\section{Lipoprotein separation}

Lipoprotein fractions were obtained from a pool of 10 sera of healthy individuals with hypertriglyceridaemia, by ultracentrifugation on a $\mathrm{NaCl}$ gradient (40). Lipoproteins were not prepared from VL patients sera because of clinical limitations in the volume of blood that can be collected from them. These fractions were dialysed against RPMI 1640 (GIBCO, Grand Island, NY, USA), pH $7 \cdot 2$, at $4^{\circ} \mathrm{C}$, for $24 \mathrm{~h}$, in sterile conditions, and stored at $-20^{\circ} \mathrm{C}$.

\section{Infection of macrophages with Leishmania infantum and their incubation with lipoproteins}

Healthy donors' PBMC were separated using Ficoll-Paque (Sigma Chemical Co., St. Louis, MO, USA) and cultured in 24-well plates in RPMI medium supplemented with 
amino acids and 10\% normal AB human serum (complete $\mathrm{RPMI}$ ), at $37^{\circ} \mathrm{C}$ and $5 \%$ of $\mathrm{CO}_{2}$. After $48 \mathrm{~h}$ of culture, the wells were washed with RPMI at $37^{\circ} \mathrm{C}$ for removal of nonadherent cells and incubated in complete RPMI for 7 days, with the same culture conditions. On the eighth day of culture, $2 \times 10^{6} \mathrm{~L}$. infantum promastigotes, in stationary phase of growth, were added to some wells in the proportion of 10 promastigotes per macrophage. The cultures were incubated again for $4 \mathrm{~h}$ and washed with RPMI for removal of Leishmania from the supernatants. LDL, HDL and VLDL fractions were then added, leading to the concentrations given in the Figure 1, both to infected and uninfected macrophages. At least $70 \%$ of the macro- phages were infected in any of the cultures to which Leishmania was added. After a 24-h incubation, the culture supernatants were collected and stored at $-20^{\circ} \mathrm{C}$.

\section{Quantification of cytokines}

Commercially available detection kits were used to determine the concentrations of IL-6, IL-12p40, TNF- $\alpha$ (Duo-set-Kit; R\&D Systems, Inc., Minneapolis, MN, USA, http://www.rndsystems.com), and IL-10 (human ELISA Set kit, BD Biosciences, Franklin Lakes, NJ, USA, http:// www.bdbiosciences.com), following the manufacturer's instructions.
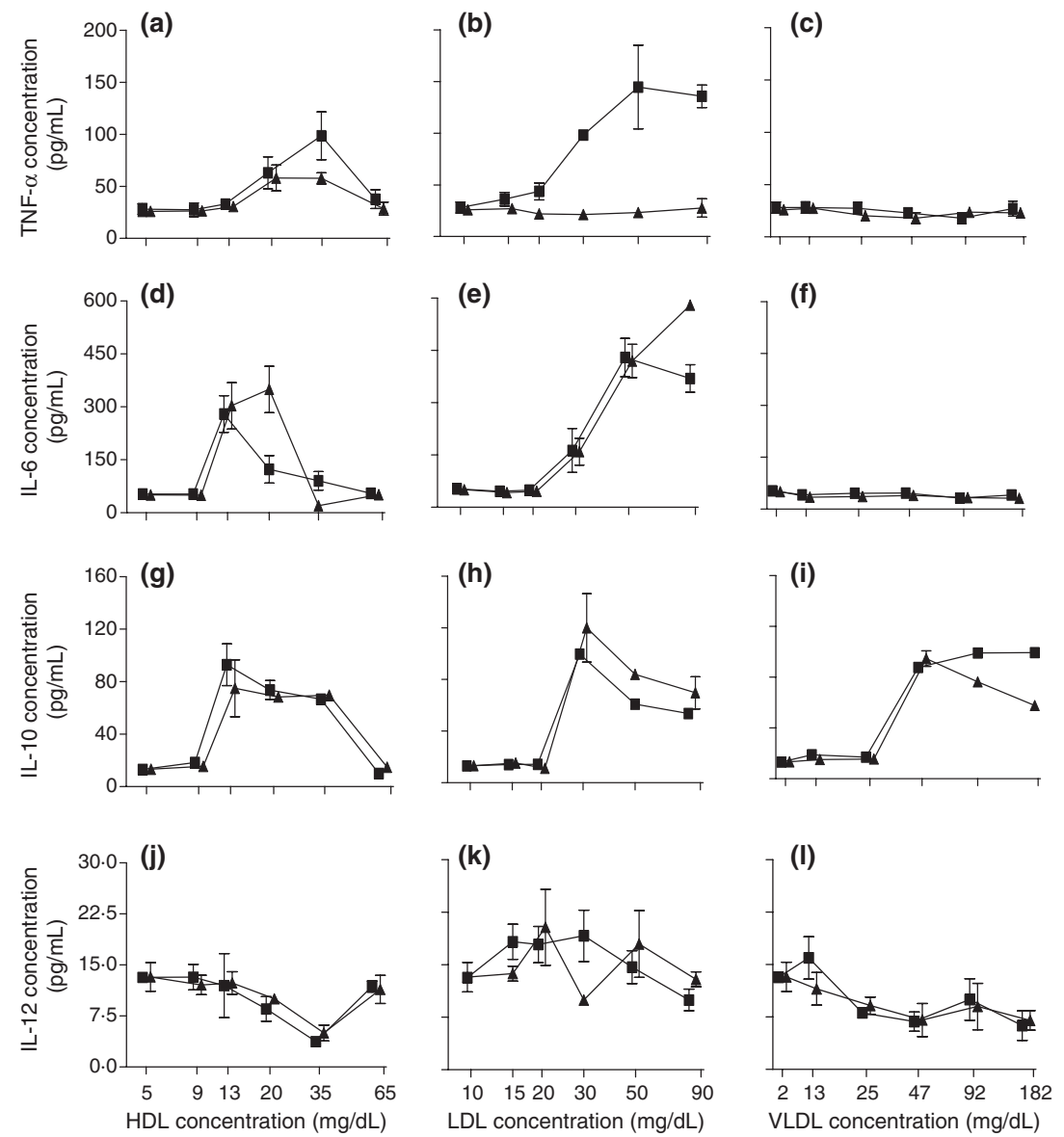

Figure 1 Concentration-response curves of the effects of LDL, HDL or VLDL on the production of TNF- $\alpha$ (a, b, c), IL-6 (d, e, f), IL-10 $(\mathrm{g}, \mathrm{h}, \mathrm{i})$ and IL-12 (j, k, l) by human monocyte-derived macrophages in vitro. The macrophages were either infected with Leishmania infantum $(\mathbf{\square})$ or uninfected $(\mathbf{\Lambda})$, as explained in the Materials and Method. Cytokine concentrations, in culture supernatants collected after $24 \mathrm{~h}$ after the addition of lipoproteins, were determined by ELISA. The data represent the mean of three experiments with cells from different donors. Vertical bars represent the standard deviations of the means. Within each experiment, each concentration of lipoprotein was assessed in triplicate. The values shown on the $x$-axis correspond to the total concentrations of each lipoprotein present in the cultures (i.e. the sums of the concentrations of the added purified lipoproteins with those of the lipoproteins that were present in the $10 \%$ normal human $\mathrm{AB}$ serum-containing culture medium; the first point on the left represents the result that was obtained when no purified lipoprotein was added). 


\section{Statistical analyses}

Analyses of the statistical significance of differences in lipidic fractions found in VL patients' sera and in normal individual sera were carried out using the paired Student's $t$-test. The statistical significance of differences in the production of cytokines by infected or uninfected macrophages, either stimulated or not stimulated with lipoproteins, was determined by using the Friedman's test and the repeated measures ANOVA.

\section{RESULTS}

\section{Analysis of lipidic profile in VL patients' sera}

High levels of triglycerides and VLDL, and low levels of apolipoprotein A1, apolipoprotein B, total cholesterol, LDL and especially HDL, were found in sera from patients with VL (Table 1). Values of HDL in these patients' sera were about six times lower than in normal individuals' sera $(P<0.05)$.

\section{Effect of lipoproteins on cytokine production by Leishmania infantum-infected macrophages}

Low density lipoprotein stimulated the production of TNF- $\alpha$ by infected PBMC-derived macrophages, and not by uninfected macrophages, starting at the concentration of $30 \mathrm{mg} / \mathrm{dL}$, and reaching a plateau with the concentration of $50 \mathrm{mg} / \mathrm{dL}(P<0.05$; Figure 1b). Although a small increase in TNF- $\alpha$ production was observed in cultures of both infected and uninfected macrophages at HDL concentrations above $13 \mathrm{mg} / \mathrm{dL}$, such difference was not statistically significant $(P>0 \cdot 05$; Figure 1a). VLDL did not stimulate the production of TNF- $\alpha$ in either infected or uninfected macrophages $(P>0.05$; Figure 1c).

The incubation with HDL and LDL, starting at the concentrations of 13 and $30 \mathrm{mg} / \mathrm{dL}$ respectively, increased IL-6 concentrations in both infected and uninfected macrophage cultures (Figure 1d, e). Addition of HDL to the cultures at concentrations higher than $20 \mathrm{mg} / \mathrm{dL}$ did not lead to increased IL-6 production. Although there was about three times more IL- 6 in uninfected than in infected macrophage cultures that contained $20 \mathrm{mg} / \mathrm{dL}$ of HDL (Figure 1d), this difference was not statistically significant $(P>0 \cdot 05)$. The addition of VLDL did not affect the concentration of IL-6 (Figure 1f).

The incubation of both infected and uninfected macrophages with concentrations equal to or above $13 \mathrm{mg} / \mathrm{dL}$ of HDL, $30 \mathrm{mg} / \mathrm{dL}$ of LDL or $47 \mathrm{mg} / \mathrm{dL}$ of VLDL led to an increase in IL-10 production $(P<0.05$; Figure $1 \mathrm{~g}, \mathrm{~h}$, i). This increase was not seen when $65 \mathrm{mg} / \mathrm{dL}$ of HDL was added to the cultures. No statistically significant differences in IL-10 concentrations between infected and uninfected macrophage cultures were observed $(P>0 \cdot 05)$.

Table 1 Lipid fractions in 26 zoonotic visceral leishmaniasis (VL) patients' and in 26 healthy individuals' sera

\begin{tabular}{|c|c|c|c|c|c|}
\hline \multirow[b]{2}{*}{ Lipid fractions } & \multirow[b]{2}{*}{$\begin{array}{l}\text { Age } \\
\text { (years) }\end{array}$} & \multirow[b]{2}{*}{$\begin{array}{l}\text { Number of } \\
\text { individuals }\end{array}$} & \multicolumn{2}{|c|}{$\begin{array}{l}\text { Concentrations of lipid fractions in } \\
\text { sera from }\end{array}$} & \multirow[b]{2}{*}{$\begin{array}{l}\text { Statistical } \\
\text { significance }^{\mathrm{a}}\end{array}$} \\
\hline & & & VL patients & $\begin{array}{l}\text { Healthy } \\
\text { individuals }\end{array}$ & \\
\hline \multirow[t]{2}{*}{ Total cholesterol } & 1-12 & 12 & $91 \pm 33^{\mathrm{b}}$ & $201 \pm 34$ & $P<0.05$ \\
\hline & $>18$ & 14 & $104 \pm 37$ & $186 \pm 24$ & $P<0.05$ \\
\hline \multirow[t]{2}{*}{ LDL } & $1-12$ & 12 & $30 \pm 13$ & $135 \pm 33$ & $P<0.05$ \\
\hline & $>18$ & 14 & $53 \pm 23$ & $113 \pm 27$ & $P<0.05$ \\
\hline \multirow[t]{2}{*}{ HDL } & 1-12 & 12 & $8 \pm 5$ & $47 \pm 17$ & $P<0.05$ \\
\hline & $>18$ & 14 & $8 \pm 6$ & $49 \pm 16$ & $P<0.05$ \\
\hline \multirow[t]{2}{*}{ VLDL } & 1-12 & 12 & $53 \pm 32$ & $19 \pm 5$ & $P<0.05$ \\
\hline & $>18$ & 14 & $43 \pm 14$ & $24 \pm 6$ & $P<0.05$ \\
\hline \multirow[t]{2}{*}{ Triglycerides } & 1-12 & 12 & $266 \pm 158$ & $96 \pm 24$ & $P<0.05$ \\
\hline & $>18$ & 14 & $216 \pm 71$ & $118 \pm 30$ & $P<0.05$ \\
\hline \multirow[t]{2}{*}{ APO A1 } & $1-12$ & 12 & $79 \pm 38$ & $188 \pm 51$ & $P<0.05$ \\
\hline & $>18$ & 14 & $80 \pm 31$ & $195 \pm 51$ & $P<0,05$ \\
\hline \multirow[t]{2}{*}{ APO B } & 1-12 & 12 & $80 \pm 25$ & $99 \pm 20$ & $P>0.05$ \\
\hline & $>18$ & 14 & $80 \pm 19$ & $117 \pm 27$ & $P<0.05$ \\
\hline
\end{tabular}

LDL, low density lipoprotein; HDL, high density lipoprotein; VLDL, very low density lipoprotein.

${ }^{a}$ Statistical significance of differences between VL patients and age-matched healthy individuals, as determined by the paired Student's $t$ test.

${ }^{\mathrm{b}}$ Mean concentration in $\mathrm{mg} / \mathrm{dL} \pm$ standard deviation of the mean. 
The lipoproteins did not significantly affect the production of IL-12 (Figure 1j, k, 1), with the exception of a reduction when the cells were incubated with $35 \mathrm{mg} / \mathrm{dL}$ of HDL (Figure 1j).

\section{DISCUSSION}

As shown herein, VL patients have high circulating levels of triglycerides and VLDL, and markedly reduced levels of total cholesterol, LDL, HDL and apolipoprotein A (which is mainly involved in the composition of HDL), and a modest decrease in apolipoprotein B (which participates in the composition of both LDL and VLDL). These results are in agreement with those published by Bertoli et al., who reported hypertriglyceridaemia in 28 patients with VL (29), by Bekaert et al., who reported decreased plasma levels of HDL, LDL, apolipoproteins A-I and A-II and increased triglycerides levels in 17 Tunisian patients with VL (28), and by Liberopoulos et al., who reported hypocholesterolaemia in a patient with VL (30). VLDL plays a role in the transfer of triglyceride and cholesterol from liver to muscular and adipose tissue. The mechanisms controlling its production are poorly understood and its acquirement by tissues is dependent upon the activity of enzymes such as endothelial lipoprotein lipase, which may be impaired in patients with VL (41). A decrease in VLDL metabolism to intermediate density lipoprotein (IDL) may contribute to the decreased LDL formation in VL patients. The low rate of hydrolysis of VLDL triglyceride by lipoprotein lipase would also decrease the availability of unesterified cholesterol, phospholipids and various lipoproteins that enter the constitution of HDL. Additionally, a reduced synthesis of apolipoprotein $\mathrm{A}$ in liver and intestine, and an enhanced activity of the LDL receptor, may be trigged by proinflammatory cytokines, such as IL-6 $(28,30,42)$.

In this study, both infected and uninfected macrophages were shown to produce IL-6 and IL-10 when incubated with physiological concentrations of HDL and LDL in vitro. The production of IL-6 and IL-10; however, was not affected by infection of the macrophages by $L$. infantum. The spontaneous production of IL- 6 and IL-10 by macrophages, in the presence of physiological concentrations of plasma lipoproteins, may reflect a real phenomenon, i.e. it would also occur in macrophages that are not stimulated in vivo, or may result from cell activation because of in vitro conditions. Increased concentrations of both IL-10 and IL- 6 have been found in the plasma of VL patients $(15,16,19)$. This work provides evidence that the simple infection of human monocyte-derived macrophages by $L$. infantum does not lead to the production of these cytokines. In the case of IL-10, this has been confirmed by studies that show that the production requires the presence of anti-Leishmania antibodies (43) or bacterial lipopolysaccharide (44), although one study reported its production in the absence of additional stimulus (45). An absence of effect of Leishmania infection on IL-10 production by macrophages is consistent with the fact that $\mathrm{T}$ cells have been found to be a source of that cytokine in VL patients (46). On the other hand, in some reports, the in vitro infection of macrophages by Leishmania was shown to promote the synthesis of IL-10, IL-6, TNF- $\alpha$ and/or their correspondent mRNA, even in the presence of subphysiological concentrations of plasma lipoproteins, i.e. the concentrations present in medium containing $10 \%$ normal serum $(45,47-50)$. This is in disagreement with the data presented herein, in which infection of human macrophages by $L$. infantum stationary phase promastigotes, in the absence of physiological concentrations of lipoproteins, did not induce the production of these cytokines. This discrepancy may be explained by differences in the Leishmania and macrophage-donor species that were used in different studies, because, with the exception of one work showing increased synthesis of IL-10, all the reports referred above describe results obtained using murine macrophages, and none of them used $L$. infantum. In fact, the expression of IL-1 by infected macrophages varies in accordance with the Leishmania species and the mouse strain used (51).

Contrasting with the findings described above for IL-6 and IL-10, physiological amounts of LDL, or even the reduced amounts found in some patients with VL, synergize with $L$. infantum infection to induce TNF- $\alpha$ production by macrophages. The synergism between infection and LDL was obligatory, neither of them alone led to the production of high concentrations of the cytokine. The results described above, therefore, may support the hypothesis that LDL is required for the production of high levels of TNF- $\alpha$ in VL. In fact, the possibility that infected macrophages, in the presence of LDL, are a major source of TNF- $\alpha$, could explain the apparently paradoxical findings of high plasma levels of TNF- $\alpha$ and low numbers of circulating TNF-containing monocytes in VL patients (19): infected splenic macrophages, and not uninfected circulating monocytes, would be the main source of TNF- $\alpha$. TNF- $\alpha$ levels may be further augmented in VL patients by the presence of increased numbers of macrophages in inflammatory infiltrates. For instance, the spleen, which is usually markedly enlarged in those patients, is populated by large numbers of infected and uninfected macrophages $(52,53)$, and is very likely the major infected organ in VL. Splenic cells are all in ample contact with plasma lipoproteins, favoured by characteristics of the splenic blood circulation. An increase in TNF- $\alpha$ 
production occurs in the course of severe forms of VL, and has been associated with disease progression (13) and lymphoid tissue disorganization $(14,54)$ indicating that, when TNF- $\alpha$ is present in high levels in VL patients, its pathogenic effect may prevail upon the benefits that would arise from its controlling intracellular parasite growth (54). On the other hand, it is shown in this work that lipid concentrations, in the range of those found in the plasma of many VL patients, and therefore in their spleens, affect the production of cytokines by macrophages in vitro. An impairment of the local immune response in the spleen could well make the difference in parasite proliferation or control. The importance of this fact in the course of the disease deserves further investigation.

It is interesting to note that none of the lipoproteins affected the production of IL-12 by infected or uninfected macrophages, indicating that lipoprotein levels do not directly influence the IL-12-dependent production of IFN$\gamma(55,56)$ in VL patients. These findings also indicate that the lipoprotein preparations were not contaminated by bacterial lipopolysaccharide (LPS), as LPS induces IL-12 production by human macrophages (57).

As shown herein, the pattern of lipoprotein concentrations that is found in VL patients (low total cholesterol, low HDL, low LDL and high VLDL levels) leads to decreased productions of TNF- $\alpha$ and IL- 6 and normal production of IL-10 by macrophages in vitro, a situation that would tend to reduce inflammation. Would this constitute a negative feedback mechanism, in which increased levels of IL-6 and/or TNF- $\alpha$ would lead to increased inflammation and decreased lipoprotein levels, which, in their turn, would increase the threshold for production of IL- 6 and TNF- $\alpha$ by macrophages, leading to a reduction in their levels and in the consequent inflammation? Consistent with the view that low cholesterol levels reduce inflammation, the results of this study may have implications for the efficacy of a suggested treatment of VL. It has been suggested that it should be of clinical advantage to reduce plasma cholesterol levels in VL patients by means of specific medications (37). The data presented herein pose some doubts on the desirability of that procedure; as cholesterol levels are already much lower in VL patients than in healthy, age-matched individuals. It would nevertheless be worthwhile to carry out prospective studies on VL patients to see if there is any correlation between plasma lipid levels and disease outcome.

In this work, the effect of lipoproteins on macrophages that had been previously infected by Leishmania promastigotes was shown. Further work would be required to find out if infection by amastigotes would produce the same results. It would also be worthwhile to investigate whether the effect of LDL on Leishmania-infected macro- phages has any degree of specificity for the parasite, or if it would also occur in the case of infection by other intracellular pathogens, or even in the case of phagocytosis of inert particles. Could LDL enhance the defense against intracellular pathogens?

It is provided herein evidence that plasma lipids could play a role in the development or maintenance of the immune response found in active VL, in so far as the presence of LDL would be required for the production of TNF- $\alpha$ by L. infantum-infected macrophages, and that the levels of lipoproteins found in some VL patients would be below the threshold required for the maximal production of TNF- $\alpha$ and of IL-6. Plasma lipid alterations in VL could therefore act as one additional factor in a very likely complex interplay of parasite-, environmental- and hostdependent factors (58-60), the net result of which is the usually irreversible progression to lethality of untreated VL patients.

\section{ACKNOWLEDGEMENTS}

We are grateful to Fabíola Nascimento da Conceição for translating the first draft of the manuscript to English and to the Conselho Nacional de Desenvolvimento Científico e Tecnológico - CNPq - for financial support.

\section{REFERENCES}

1 Mauricio IL, Howard MK, Stothard JR \& Miles MA. Genomic diversity in the Leishmania donovani complex. Parasitology 1999; 119(Pt 3): 237-246.

2 Andrade TM, Carvalho EM \& Rocha H. Bacterial infections in patients with visceral leishmaniasis. J Infect Dis 1990; 162: 1354-1359.

3 Ho M, Koech DK, Iha DW \& Bryceson AD. Immunosuppression in Kenyan visceral leishmaniasis. Clin Exp Immunol 1983; 51: 207-214.

4 Ho JL, Badaro R, Schwartz A, et al. Diminished in vitro production of interleukin-1 and tumor necrosis factor-alpha during acute visceral leishmaniasis and recovery after therapy. J Infect Dis 1992; 165: 1094-1102.

5 Cillari E, Liew FY, Lo Campo P, Milano S, Mansueto S \& Salerno A. Suppression of IL-2 production by cryopreserved peripheral blood mononuclear cells from patients with active visceral leishmaniasis in Sicily. J Immunol 1988; 140: 27212726.

6 Mauricio IL, Stothard JR \& Miles MA. The strange case of Leishmania chagasi. Parasitol Today 2000; 16: 188-189.

7 Bauzer LG, Souza NA, Maingon RD \& Peixoto AA. Lutzomyia longipalpis in Brazil: a complex or a single species? A mini-review. Mem Inst Oswaldo Cruz 2007; 102: 1-12.

8 Rezai HR, Ardehali SM, Amirhakimi G \& Kharazmi A. Immunological features of kala-azar. Am J Trop Med Hyg 1978; 27: 1079-1083.

9 Carvalho EM, Badaro R, Reed SG, Jones TC \& Johnson WD Jr. Absence of gamma interferon and interleukin 2 production 
during active visceral leishmaniasis. J Clin Invest 1985; 76: 2066-2069.

10 Carvalho EM, Barral A, Pedral-Sampaio D, et al. Immunologic markers of clinical evolution in children recently infected with Leishmania donovani chagasi. J Infect Dis 1992; 165: 535540.

11 Kurkjian KM, Mahmutovic AJ, Kellar KL, Haque R, Bern C $\&$ Secor WE. Multiplex analysis of circulating cytokines in the sera of patients with different clinical forms of visceral leishmaniasis. Cytometry A 2006; 69: 353-358.

12 Sanal O, Turkkani G, Gumruk F, et al. A case of interleukin12 receptor beta-1 deficiency with recurrent leishmaniasis. Pediatr Infect Dis $J$ 2007; 26: 366-368.

13 Barral-Netto M, Badaro R, Barral A, et al. Tumor necrosis factor (cachectin) in human visceral leishmaniasis. $J$ Infect Dis 1991; 163: 853-857.

14 Engwerda CR, Ato M, Cotterell SE, et al. A role for tumor necrosis factor-alpha in remodeling the splenic marginal zone during Leishmania donovani infection. Am J Pathol 2002; 161: 429-437.

15 Caldas A, Favali C, Aquino D, et al. Balance of IL-10 and interferon-gamma plasma levels in human visceral leishmaniasis: implications in the pathogenesis. BMC Infect Dis 2005; 5: 113.

16 Ghalib HW, Piuvezam MR, Skeiky YA, et al. Interleukin 10 production correlates with pathology in human Leishmania donovani infections. J Clin Invest 1993; 92: 324-329.

17 Bacellar O, D'Oliveira A Jr, Jeronimo S \& Carvalho EM. IL-10 and IL-12 are the main regulatory cytokines in visceral leishmaniasis. Cytokine 2000; 12: 1228-1231.

18 Murphy ML, Wille U, Villegas EN, Hunter CA \& Farrell JP. IL-10 mediates susceptibility to Leishmania donovani infection. Eur J Immunol 2001; 31: 2848-2856.

19 Peruhype-Magalhaes V, Martins-Filho OA, Prata A, et al. Mixed inflammatory/regulatory cytokine profile marked by simultaneous raise of interferon-gamma and interleukin-10 and low frequency of tumour necrosis factor-alpha $(+)$ monocytes are hallmarks of active human visceral leishmaniasis due to Leishmania chagasi infection. Clin Exp Immunol 2006; 146: 124-132.

20 Chisari FV. Immunoregulatory properties of human plasma in very low density lipoproteins. J Immunol 1977; 119: 2129-2136.

21 Soares NM, Ferraz TP, Nascimento EG, Carvalho EM \& Pontes-de-Carvalho L. The major circulating immunosuppressive activity in American visceral leishmaniasis patients is associated with a high-molecular weight fraction and is not mediated by $\mathrm{IgG}, \mathrm{IgG}$ immune complexes or lipoproteins. Microb Pathog 2006; 40: 254-260.

22 Girona J, La Ville AE, Heras M, Olive S \& Masana L. Oxidized lipoproteins including HDL and their lipid peroxidation products inhibit TNF-alpha secretion by THP-1 human macrophages. Free Radic Biol Med 1997; 23: 658-667.

23 Fessel WJ, Follansbee SE \& Rego J. High-density lipoprotein cholesterol is low in HIV-infected patients with lipodystrophic fat expansions: implications for pathogenesis of fat redistribution. AIDS 2002; 16: 1785-1789.

24 Fernandez-Miranda C, Pulido F, Carrillo JL, et al. Lipoprotein alterations in patients with HIV infection: relation with cellular and humoral immune markers. Clin Chim Acta 1998; 274: 63-70.

25 Ramos TM, de Vasconcelos AS, de Carvalho VC \& Lima VL. Alterations in cholesterol, triglyceride and total phospholipid levels in plasma of Callithrix jacchus (sagui) reinfected by Schistosoma mansoni. Rev Soc Bras Med Trop 2004; 37: 37-40.

26 Nieto CG, Barrera R, Habela MA, et al. Changes in the plasma concentrations of lipids and lipoprotein fractions in dogs infected with Leishmania infantum. Vet Parasitol 1992; 44: $175-182$

27 Bekaert ED, Dole E, Dubois DY, et al. Alterations in lipoprotein density classes in infantile visceral leishmaniasis: presence of apolipoprotein SAA. Eur J Clin Invest 1992; 22: 190-199.

28 Bekaert ED, Kallel R, Bouma ME, et al. Plasma lipoproteins in infantile visceral leishmaniasis: deficiency of apolipoproteins A-I and A-II. Clin Chim Acta 1989; 184: 181-191.

29 Bertoli A, Greco AV, Caputo S, Caradonna P, Grieco A \& Laghi V. Visceral leishmaniasis presenting with hypertrigliceridaemia. Lancet 1982; 2: 504-505.

30 Liberopoulos E, Alexandridis G, Bairaktari E \& Elisaf M. Severe hypocholesterolemia with reduced serum lipoprotein(a) in a patient with visceral leishmaniasis. Ann Clin Lab Sci 2002; 32: $305-308$

31 Gatfield J \& Pieters J. Essential role for cholesterol in entry of mycobacteria into macrophages. Science 2000; 288: 1647-1650.

32 Lauer S, VanWye J, Harrison T, et al. Vacuolar uptake of host components, and a role for cholesterol and sphingomyelin in malarial infection. EMBO J 2000; 19: 3556-3564.

33 Jutras I, Abrami L \& Dautry-Varsat A. Entry of the lymphogranuloma venereum strain of Chlamydia trachomatis into host cells involves cholesterol-rich membrane domains. Infect Immun 2003; 71: 260-266.

34 Seveau S, Bierne H, Giroux S, Prevost MC \& Cossart P. Role of lipid rafts in E-cadherin - and HGF-R/Met - mediated entry of Listeria monocytogenes into host cells. J Cell Biol 2004; 166: 743-753.

35 Tewary P, Veena K, Pucadyil TJ, Chattopadhyay A \& Madhubala $\mathrm{R}$. The sterol-binding antibiotic nystatin inhibits entry of non-opsonized Leishmania donovani into macrophages. Biochem Biophys Res Commun 2006; 339: 661-666.

36 Rodriguez NE, Gaur U \& Wilson ME. Role of caveolae in Leishmania chagasi phagocytosis and intracellular survival in macrophages. Cell Microbiol 2006; 8: 1106-1120.

37 Pucadyil TJ \& Chattopadhyay A. Cholesterol: a potential therapeutic target in Leishmania infection? Trends Parasitol 2007; 23: 49-53.

38 Chakraborty D, Banerjee S, Sen A, Banerjee KK, Das P \& Roy S. Leishmania donovani affects antigen presentation of macrophage by disrupting lipid rafts. J Immunol 2005; 175: 3214-3224.

39 Voller A, Bartlett A \& Bidwell DE. Enzyme immunoassays with special reference to ELISA techniques. J Clin Pathol 1978; 31: 507-520.

40 Redgrave TG, Roberts DC \& West CE. Separation of plasma lipoproteins by density-gradient ultracentrifugation. Anal Biochem 1975; 65: 42-49.

41 Nanjee MN, Cooke CJ, Olszewski WL \& Miller NE. Lipid and apolipoprotein concentrations in prenodal leg lymph of fasted humans. Associations with plasma concentrations in normal subjects, lipoprotein lipase deficiency, and LCAT deficiency. J Lipid Res 2000; 41: 1317-1327.

42 Kallel R, Bekaert ED, Dubois DY, Alcindor LG, AyraultJarrier M \& Mebazza A. Acute phase proteins and plasma lipoproteins during antimony treatment in infantile visceral leishmaniasis. Clin Physiol Biochem 1993; 10: 8-12.

43 Miles SA, Conrad SM, Alves RG, Jeronimo SM \& Mosser DM. A role for IgG immune complexes during infection with 
the intracellular pathogen Leishmania. $J$ Exp Med 2005; 201: 747-754.

44 Meddeb-Garnaoui A, Zrelli H \& Dellagi K. Effects of tropism and virulence of Leishmania parasites on cytokine production by infected human monocytes. Clin Exp Immunol 2009; 155: 199-206.

45 Chandra D \& Naik S. Leishmania donovani infection downregulates TLR2-stimulated IL-12p40 and activates IL-10 in cells of macrophage/monocytic lineage by modulating MAPK pathways through a contact-dependent mechanism. Clin Exp Immunol 2008; 154: 224-234.

46 Nylen S, Maurya R, Eidsmo L, Manandhar KD, Sundar S \& Sacks D. Splenic accumulation of IL-10 mRNA in T cells distinct from CD4+CD25+ (Foxp3) regulatory $\mathrm{T}$ cells in human visceral leishmaniasis. $J$ Exp Med 2007; 204: 805-817.

47 Green SJ, Mellouk S, Hoffman SL, Meltzer MS \& Nacy CA. Cellular mechanisms of nonspecific immunity to intracellular infection: cytokine-induced synthesis of toxic nitrogen oxides from L-arginine by macrophages and hepatocytes. Immunol Lett 1990; 25: 15-19.

48 Theodos CM, Povinelli L, Molina R, Sherry B \& Titus RG. Role of tumor necrosis factor in macrophage leishmanicidal activity in vitro and resistance to cutaneous leishmaniasis in vivo. Infect Immun 1991; 59: 2839-2842.

49 Moore KJ \& Matlashewski G. Intracellular infection by Leishmania donovani inhibits macrophage apoptosis. J Immunol 1994; 152: 2930-2937.

50 Gorak PM, Engwerda CR \& Kaye PM. Dendritic cells, but not macrophages, produce IL-12 immediately following Leishmania donovani infection. Eur J Immunol 1998; 28: 687-695.

51 Delfino D, Chiofalo MS, Riggio G, et al. Induction of interleukin 1 alpha in murine macrophages infected in vitro with different species and strains of Leishmania. Microb Pathog 1995; 18: 73-80.

52 Veress B, Omer A, Satir AA \& El Hassan AM. Morphology of the spleen and lymph nodes in fatal visceral leishmaniasis. Immunology 1977; 33: 605-610.
53 Chulay JD \& Bryceson AD. Quantitation of amastigotes of Leishmania donovani in smears of splenic aspirates from patients with visceral leishmaniasis. Am J Trop Med Hyg 1983; 32: 475-479.

54 Engwerda CR, Ato M \& Kaye PM. Macrophages, pathology and parasite persistence in experimental visceral leishmaniasis. Trends Parasitol 2004; 20: 524-530.

55 Chan SH, Kobayashi M, Santoli D, Perussia B \& Trinchieri G. Mechanisms of IFN-gamma induction by natural killer cell stimulatory factor (NKSF/IL-12). Role of transcription and mRNA stability in the synergistic interaction between NKSF and IL-2. J Immunol 1992; 148: 92-98.

56 Manetti R, Gerosa F, Giudizi MG, et al. Interleukin 12 induces stable priming for interferon gamma (IFN-gamma) production during differentiation of human $\mathrm{T}$ helper (Th) cells and transient IFN-gamma production in established Th2 cell clones. J Exp Med 1994; 179: 1273-1283.

57 Nau GJ, Schlesinger A, Richmond JF \& Young RA. Cumulative Toll-like receptor activation in human macrophages treated with whole bacteria. J Immunol 2003; 170: 5203-5209.

58 Van Weyenbergh J, Santana G, D'Oliveira A Jr, et al. Zinc/copper imbalance reflects immune dysfunction in human leishmaniasis: an ex vivo and in vitro study. BMC Infect Dis 2004; 4: 50.

59 Rosa R, Rodrigues OR, Marques C \& Santos-Gomes GM. Leishmania infantum: soluble proteins released by the parasite exert differential effects on host immune response. Exp Parasitol 2005; 109: 106-114.

60 Jeronimo SM, Holst AK, Jamieson SE, et al. Genes at human chromosome $5 \mathrm{q} 31.1$ regulate delayed-type hypersensitivity responses associated with Leishmania chagasi infection. Genes Immun 2007; 8: 539-551. 